\title{
Prevalence of syphilis infection in different tiers of female sex workers in China: implications for surveillance and interventions
}

Xiang-Sheng Chen ${ }^{1 *}$, Qian-Qiu Wang ${ }^{1}$, Yue-Ping Yin', Guo-Jun Liang ${ }^{1}$, Ning Jiang ${ }^{1}$, Li-Gang Yang ${ }^{2}$, Qiao Liu ${ }^{3}$, Yu-Jiao Zhou ${ }^{4}$, Xi-Ping Huan ${ }^{5}$, Wan-Hui Wei ${ }^{1}$ and Baoxi Wang ${ }^{1}$

\begin{abstract}
Background: Syphilis has made a dramatic resurgence in China during the past two decades and become the third most prevalent notifiable infectious disease in China. Female sex workers (FSWs) have become one of key populations for the epidemic. In order to investigate syphilis infection among different tiers of FSWs, a crosssectional study was conducted in 8 sites in China.

Methods: Serum specimens ( $n=7,118$ ) were collected to test for syphilis and questionnaire interviews were conducted to obtain socio-demographic and behavioral information among FSWs recruited from different types of venues. FSWs were categorized into three tiers (high-, middle- and low-tier FSWs) based on the venues where they solicited clients. Serum specimens were screened with enzyme-linked immunosorbent assay (ELISA) for treponemal antibody followed by confirmation with non-treponemal toluidine red unheated serum test (TRUST) for positive ELISA specimens to determine syphilis infection. A logistic regression model was used to determine factors associated with syphilis infection.
\end{abstract}

Results: Overall syphilis prevalence was 5.0\% (95\%Cl, 4.5-5.5\%). Low-tier FSWs had the highest prevalence (9.7\%; $95 \% \mathrm{Cl}, 8.3-11.1 \%)$, followed by middle-tier $(4.3 \%$; $95 \% \mathrm{Cl}, 3.6-5.0 \%, P<0.001)$ and high-tier FSWs $(2.2 \% ; 95 \% \mathrm{Cl}, 1.6-$ $2.9 \%, P<0.001)$. Factors independently associated with syphilis infection included older age, lower education level, geographic location, lower tier of typology, and injection drug use.

Conclusions: This multi-site survey showed a high prevalence of syphilis infection among FSWs and substantial disparities in syphilis prevalence by the tier of FSWs. The difference in syphilis prevalence is substantial between different tiers of FSWs, with the highest rate among low-tier FSWs. Thus, current surveillance and intervention activities, which have low coverage in low-tier FSWs in China, should be further examined.

Keywords: Syphilis, Prevalence, Typology, Female sex worker, China

\section{Background}

Syphilis has made a dramatic resurgence in China during the past two decades [1]. Heterosexual contacts have become one of primary modes of HIV transmission in China [2,3]. Syphilis has been considered as one of factors to facilitate HIV transmission [4]. Female sex workers (FSWs) are more at risk of having HIV, syphilis and

\footnotetext{
* Correspondence: chenxs@ncstdlc.org

'National Center for STD Control, Chinese Academy of Medical Sciences and Peking Union Medical College Institute of Dermatology, 12 Jiangwangmiao Street, Nanjing 210042, China

Full list of author information is available at the end of the article
}

other sexually transmitted infections (STIs). Syphilis has been ranked as the third most prevalent notifiable infectious disease in China. Thus, the syphilis epidemic holds important relevance for infectious disease burden control in China and the region. Assessing the disease burden of syphilis among FSWs is necessary for planning primary and secondary prevention control measures. Syphilis surveillance in China has relied largely on case reports submitted by hospitals or clinics through a website-based case-reporting system [1]. Syphilis prevalence monitoring among specific high-risk populations including FSWs is conducted through HIV sentinel 
surveillance surveys to provide trends for gauging the effectiveness of prevention efforts [5]. The reported incidence of primary and secondary syphilis (reflecting the intensity of recent transmission) was 11.7 cases per 100 , 000 residents in 2009, which had increased by 2.1 times since 2005 [6]. However, case report data may underestimate the true burden of syphilis due to inadequate screening among asymptomatic infected persons and underreporting of the diagnosed cases. Current sentinel surveillance for HIV and syphilis infections among FSWs in China is usually based on recruitment of a convenience sample of FSWs working in entertainment establishments. Based on a list of establishments which are typically used for interventions through outreach work, some venues are selected for recruiting a convenience sample of FSWs for a questionnaire survey and specimen collection. Freelance FSWs are usually not included in the surveillance of most sentinel sites. In this case, the FSWs working at luxurious sex venues (e. g. karaoke bars or hotels) are usually disproportionately recruited. In this paper, we present a multi-site epidemiological study on the prevalence and risk factors of syphilis among FSWs recruited from different typologies of venues in China, and discuss the implications of these findings for surveillance and interventions.

\section{Methods}

\section{Study sites and survey design}

The study was conducted in eight cities in the eastern part (Changzhou and Yangzhou in Jiangsu Province) and southern part (Hezhou and Wuzhou in Guangxi Province, Jiangmen and Maoming in Guangdong Province, and Sanya and Qionghai in Hainan Province) of China. We selected cities to encompass areas where STIs are highly prevalent and where sexual contact is the main route transmission of HIV in China.

The Mega Projects were the China Ministry of Science and Technology (MOST) and Ministry of Health $(\mathrm{MOH})$ projects awarded to pursue the most important public health issues in China. The current study was a baseline survey (a cross-sectional study) for one of the Mega Projects to create cohorts for analyzing the impact of expanded STI care on new HIV infection rate among high-risk groups. FSW settings were mapped and categorized in each study site and a convenience sample design was used to recruit a sample of the FSWs. Each participant was interviewed using a structured questionnaire to collect data on socio-demographic and behavioral information, and then underwent a biologic specimen collection.

\section{Participants}

Between June and September 2009, venues where FSWs solicited clients were mapped and categorized in each study site, and potential participants were identified at the different categories of venues. FSWs were recruited by outreach workers and eligibility requirements included age $>16$ years (to encompass FSWs representative of those at risk for STIs); ability to give consent; and having provided commercial sex in sex-work venues or rented apartments for money or goods within the previous three months. FSWs were classified into three subgroups based on the different categories of venues where FSWs solicited clients, i.e. high-tier FSWs (HTFSWs), middle-tier FSWs (MT-FSWs) and low-tier FSWs (LT-FSWs). High-tier group included FSWs who solicited in karaoke bars, or hotels; middle-tier group included FSWs who solicited in hair salons or barber shops, massage parlors, foot bathing shops, roadside shops, guesthouses, or roadside restaurants; and low-tier group included FSWs who solicited on the street or public outdoor places. MT-FSWs were sampled at a higher rate compared with the HT-FSW, and all accessible and acceptable LT-FSWs were recruited to allow sufficient sample sizes for analysis.

Site staff secured verbal consent from subjects to have blood drawn for free syphilis and HIV testing and to be interviewed with an anonymous and structured questionnaire by the trained outreach workers. All participants tested for syphilis and HIV were informed of their syphilis test results by an outreach team member within a week and informed of their HIV results a few weeks later. Participants with positive tests received counseling messages and were referred to designated clinics for further evaluation and possible treatment according to the national guidelines. No unique identifiers were obtained. A small gift priced at 30 yuan (around 5 US dollars) as an incentive was given to those women who agreed to participate in the survey.

The study protocols were reviewed and approved by the Medical Ethics Committee of the Chinese Academy of Medical Sciences Institute of Dermatology and National Center for Sexually Transmitted Disease Control in Nanjing.

\section{Laboratory methods}

Sera from the participants were evaluated at the STI laboratories of local CDCs or institutes of dermatology and venereology using serologic tests for determining syphilis infection according to the national algorithms. All specimens were first screened for treponemal antibody using an enzyme-linked immunosorbent assay (ELISA). Specimens with positive ELISA underwent a qualitative non-treponemal toluidine red unheated serum test (TRUST) testing, and those specimens with reactive TRUST further underwent a quantitative/titer TRUST testing. For calculating the prevalence of syphilis, the definition of syphilis infection included that 
specimens must have a positive ELISA and a positive TRUST [7].

Performance of the tests was evaluated on internal quality assurance procedures through re-testing all the serologically positive specimens and $10 \%$ of the negative specimens. The tests were found to have a favorable performance in the study sites.

\section{Statistical analysis}

All data from questionnaires, and laboratory results were concurrently double-entered into a computer database using EpiData Software (version 3.0) by independent research assistants, and databases were evaluated for congruency. When database entries conflicted, the original test results and surveys for these cases were retrieved to correct entered data. Prevalence of syphilis infection, with $95 \%$ confidence intervals (CIs), was measured. We used univariate and multivariate logistic regression to evaluate factors associated with syphilis infection using backward stepwise elimination. The variables attaining $p<0.10$ significance in univariate analysis were included in the multivariate regression analysis, retaining only variables achieving $p<0.05$ significance in the final model. Odds ratios (ORs), with 95\% CIs, for risk factors for acquiring the infection were also determined. All statistical analysis was performed using Statistical Program for Social Sciences (SPSS, version 13.0, Chicago, IL) software.

\section{Results}

A total of 7,118 FSWs, including 2,144 (30.1\%) HTFSWs, 3,227 (45.3\%) MT-FSWs and 1,747 (24.5\%) were enrolled from eight study sites to participate in a baseline survey during June and September 2009. The rates to refuse participating in the study were $8-12 \%$ in HTFSWs, $20-25 \%$ in MT-FSWs and $30-35 \%$ in LT-FSWs. Overall, 7,118 specimens underwent syphilis ELISA testing followed by TRUST testing for those with positive ELISA. The prevalence of syphilis infection in the study population was $5.0 \%$ (95\% CI, $4.5 \%-5.5 \%)$.

Table 1 shows syphilis prevalence by selected sociodemographic and behavioral characteristics. In univariate analysis, the prevalence increased with older age and less education $(P<0.001$ for each). FSWs in Guangxi had a higher prevalence $(7.1 \%$; 95\% CI, $6.1-8.1 \%)$ than those from other 3 provinces. Within Guangxi, Hezhou had the highest prevalence (12.2\%; 95\%CI. 10.0-14.5\%). Those FSWs who were separated, divorced or windowed in marital status had a higher prevalence $(9.9 \%$; $95 \% \mathrm{CI}$, $5.8-14.0 \%)$ than those who were married $(7.0 \% ; 95 \% \mathrm{CI}$, 6.0-8.0\%) or never married (3.5\%; 95\%CI, 3.0-4.1\%). Syphilis prevalence was highest among LT-FSWs (9.7\%; 95\%CI, 8.3-11.1\%), followed by MT-FSWs (4.3\%; 95\%CI, 3.6-5.0\%, $P<0.001)$ and HT-FSWs $(2.2 \%$; 95\%CI, 1.6-
2.9, $P<0.001)$. Syphilis prevalence was also higher among persons with a positive HIV test $(14.0 \%$; $95 \% \mathrm{CI}$, 3.6-24.3\%) than those with a negative HIV test (4.9\%; 95\%CI, 4.4-5.5; $P=0.007$ ). A small number of FSWs reported a history of needle-sharing, which limited our ability to make reliable estimates of syphilis prevalence among FSWs who shared needles. However, the prevalence was higher among FSWs who reported injecting drugs (25.0\%; 95\%CI, 6.0-44.0\%) than those who did not (4.9\%; 95\%CI, 4.4-5.4\%, $P<0.001)$. The difference in syphilis prevalence between FSWs who reported consistent use of a condom and no use of a condom in the past month was not statistically significant $(P=0.257)$.

In multivariate logistic regression analysis (Table 2), the factors associated with syphilis prevalence were older age (AOR $>2.62$ as compared with the age group of $16-19$ years, $P<0.001$ ), study site rather than Yangzhou site (AOR $>4.17$ as compared with the Yangzhou site, $P<0.001$ ), lower education level (AOR $>1.56$ as compared with senior high school or above, $P<0.03$ ), lower tier in FSW typology (AOR > 1.68 as compared with high-tier FSWs, $P<0.008)$, and injection drug use $($ AOR $=6.17$ as compared with no injection drug use, $P$ $=0.001)$. Multivariate logistic regression analysis showed that the LT-FSWs tended to be older with age of 40 years or above $(\mathrm{AOR}=1.81$ as compared with age of 16-19 years, $P<0.001$ ), less education level (AOR > 2.54 as compared with senior high school or above, $P<$ 0.001 ), married or separated/divorced/widowed status $(\mathrm{AOR}=1.86$ as compared with non-married status, $P<$ 0.001 ), non-Han minority (AOR $=1.29$ as compared with Han minority, $P=0.005$ ), and local residence (AOR $=2.11$ as compared with non-local residence, $P<$ $0.001)$, Table 3.

\section{Discussion}

Commercial sex is illegal in China. By 2005, the Chinese Center for Disease Control and Prevention (CDC) estimated that there were 2.8 to 4.5 million FSWs nationwide [8]. Risk behaviors and socio-demographic characteristics of FSWs linked to the sex trade may be associated with risk of STI infection, health care seeking behaviors, and/or social stigma $[9,10]$. There have been several ways to classify women who provide sex for money or gifts. A study has categorized FSWs into seven typologies according to their workplace and organization, income and demographics, and approximate sexual risk [11], while other studies have categorized them into 3 tiers simply based on sex work venues and socio-economic contexts $[12,13]$. Despite the fact that no consensus on FSW typologies has been met in China, typology of FSWs is important for design of surveillance and intervention programs. 
Table 1 Prevalence of syphilis by demographic and behavioral characteristics and HIV infection (univariate analysis)

\begin{tabular}{|c|c|c|c|c|}
\hline Characteristics & Number* & $\begin{array}{l}\text { Prevalence } \\
\%(95 \% \mathrm{Cl})\end{array}$ & $P$ value & OR $(95 \% \mathrm{Cl})$ \\
\hline Total & 7118 & $5.0(4.5-5.5)$ & & \\
\hline Age in years (median, interquartile range) & $24(16-56)$ & & $<0.001$ & $1.08(1.07,1.10)$ \\
\hline Age group & & & $<0.001$ & \\
\hline $16-19$ & 1015 & $1.7(1.0,2.5)$ & & 1.0 (Reference) \\
\hline $20-29$ & 4197 & $4.0(3.0,4.6)$ & & $2.42(1.46,4.00)$ \\
\hline 30-39 & 1519 & $7.8(6.0,9.2)$ & & $4.98(2.97,8.33)$ \\
\hline $40-$ & 387 & $14.4(11.0,18.0)$ & & $9.85(5.63,17.23)$ \\
\hline Province & & & $<0.001$ & \\
\hline Jiangsu & 1791 & $3.6(2.8-4.5)$ & & 1.0 (Reference) \\
\hline Guangdong & 1361 & $4.0(2.9-5.0)$ & & $1.10(0.76,1.59)$ \\
\hline Guangxi & 2515 & $7.1(6.1-8.1)$ & & $2.03(1.52,2.72)$ \\
\hline Hainan & 1451 & $3.9(2.9-4.9)$ & & $1.10(0.77,1.59)$ \\
\hline Site city & & & $<0.001$ & \\
\hline Yangzhou & 929 & $0.8(0.2-1.3)$ & & 1.0 (Reference) \\
\hline Changzhou & 862 & $6.7(5.1-8.4)$ & & $9.49(4.31,20.92)$ \\
\hline Jiangmen & 533 & $3.0(1.6-4.6)$ & & $4.07(1.66,9.95)$ \\
\hline Maoming & 828 & $4.6(3.2-6.0)$ & & $6.39(2.84,14.38)$ \\
\hline Wuzhou & 1706 & $4.7(3.7-5.7)$ & & $6.49(2.99,14.11)$ \\
\hline Hezhou & 809 & $12.2(10.0-14.5)$ & & $18.33(8.46,39.69)$ \\
\hline Qionghai & 643 & $4.4(2.8-5.9)$ & & $6.25(2.71,14.40)$ \\
\hline Sanya & 808 & $3.6(2.3-4.9)$ & & $4.89(2.13,11.23)$ \\
\hline Education & & & $<0.001$ & \\
\hline Primary or less & 1140 & $9.4(7.7-11.1)$ & & $4.42(2.91,6.72)$ \\
\hline Junior high school & 4727 & $4.6(4.0-5.2)$ & & $2.04(1.38,3.02)$ \\
\hline Senior high school and above & 1243 & $2.3(1.5-3.2)$ & & 1.0 (Reference) \\
\hline Marital status & & & $<0.001$ & \\
\hline Never married & 4262 & $3.5(3.0-4.1)$ & & 1.0 (Reference) \\
\hline Married & 2641 & $7.0(6.0-8.0)$ & & $2.08(1.67,2.59)$ \\
\hline Separated/divorced/widowed & 202 & $9.9(5.8-14.0)$ & & $3.03(1.86,4.95)$ \\
\hline Ethnicity & & & 0.452 & \\
\hline Han & 6263 & $5.0(4.5-5.5)$ & & 1.0 (Reference) \\
\hline Minority & 827 & $5.6(4.0-7.2)$ & & $1.13(0.82,1.55)$ \\
\hline Household registration(hukou) & & & 0.322 & \\
\hline Local & 1859 & $4.6(3.7-5.6)$ & & 1.0 (Reference) \\
\hline Not local & 5246 & $5.2(4.6-5.8)$ & & $1.13(0.88,1.46)$ \\
\hline Typology & & & $<0.001$ & \\
\hline High-tier & 2144 & $2.2(1.6-2.9)$ & & 1.0 (Reference) \\
\hline Middle-tier & 3227 & $4.3(3.6-5.0)$ & & $1.95(1.40,2.73)$ \\
\hline Low-tier & 1747 & $9.7(8.3-11.1)$ & & $4.76(3.43,6.60)$ \\
\hline Having had any STI symptoms past year & & & 0.658 & \\
\hline Yes & 2892 & $4.9(4.1-5.7)$ & & $0.95(0.77,1.18)$ \\
\hline No & 4200 & $5.1(4.4-5.8)$ & & 1.0 (Reference) \\
\hline Consistent condom use in past month & & & 0.254 & \\
\hline Yes & 3282 & $5.4(4.7-6.2)$ & & $1.14(0.91,1.42)$ \\
\hline No & 3266 & $4.8(4.0-5.5)$ & & 1.0 (Reference) \\
\hline Injecting drug use & & & $<0.001$ & \\
\hline Yes & 20 & $25.0(6.0-44.0)$ & & $6.39(2.31,17.69)$ \\
\hline No & 7063 & $4.9(4.4-5.4)$ & & 1.0 (Reference) \\
\hline HIV infection & & & 0.007 & \\
\hline Positive & 43 & $14.0(3.6-24.3)$ & & $3.11(1.30,7.41)$ \\
\hline Negative & 7059 & $4.9(4.4-5.5)$ & & 1.0 (Reference) \\
\hline
\end{tabular}


Table 2 Multivariate analysis of factors associated with syphilis infection

\begin{tabular}{ccc}
\hline Factor & Adjusted OR (95\%Cl) & $\mathbf{P}$ \\
\hline Age group in years & 1.0 (Reference) & - \\
$16-19$ & $2.62(1.57,4.36)$ & $<0.001$ \\
$20-29$ & $4.37(2.57,7.42)$ & $<0.001$ \\
$30-39$ & $7.81(4.36,14.01)$ & $<0.001$ \\
$40-$ & & \\
Site city & 1.0 (Reference) & - \\
Yangzhou & $14.35(6.43,32.03)$ & $<0.001$ \\
Changzhou & $9.51(3.76,24.08)$ & $<0.001$ \\
Jiangmen & $10.62(4.61,24.47)$ & $<0.001$ \\
Maoming & $4.17(1.90,9.17)$ & $<0.001$ \\
Wuzhou & $13.54(6.17,29.71)$ & $<0.001$ \\
Hezhou & $9.44(4.00,22.29)$ & $<0.001$ \\
Qionghai & $8.14(3.48,19.02)$ & $<0.001$ \\
Sanya & & \\
Education & $2.10(1.34,3.30)$ & 0.001 \\
Primary or less & $1.56(1.04,2.35)$ & 0.031 \\
Junior high school & $1.0($ Reference $)$ & - \\
Senior high school and above & & - \\
Typology & $1.0($ Reference) & - \\
High-tier & $1.68(1.15,2.46)$ & 0.008 \\
Middle-tier & $4.05(2.60,6.31)$ & $<0.001$ \\
Low-tier & & 0.001 \\
Injecting drug use & $1.0($ Reference $)$ & - \\
Yes & & \\
No & & \\
\hline
\end{tabular}

$O R$ odds ratio, $\mathrm{Cl}$ confidence interval.

A substantial prevalence of syphilis was found among FSWs in the study areas. These findings are consistent with the results reported elsewhere among FSWs with $5.3 \%$ in Sichuan [14], and 3\% in Shandong [15], but are much higher than the median level of national prevalence $(0.5-1.8 \%)$ [5]. The prevalence varies significantly by different tier of FSWs, showing a higher prevalence among LT-FSWs (9.7\%) than MT-FSWs (4.3\%) or HTFSWs (2.2\%). A systematic review of published studies found that low-tier FSWs are approximately twice as likely to have syphilis [16]. Some studies have found extremely high syphilis prevalence in this group, ranging from $10-38 \%[13,17]$. Studies have shown that the FSWs working in low-tier venues tended to have greater number of clients, but infrequently used condoms due to extra payment for unsafe sex [17-19]. Low-tier FSWs either independently solicit clients on streets (streetwalking FSWs) or find clients (some knew each other) through nearby construction sites and factories [11]. Our results further indicate that older women are more likely to be infected with syphilis, and the women with less education are at a greater risk of infection. A 2006 national probability survey among men $15-49$ years old
Table 3 Multivariate analysis of factors associated with low FSW typology

\begin{tabular}{|c|c|c|}
\hline Factor & $\begin{array}{l}\text { Low vs. middle/high tiers } \\
\text { AOR, } 95 \% \mathrm{Cl}\end{array}$ & $P$ value \\
\hline \multicolumn{3}{|l|}{ Age group in years } \\
\hline $16-19$ & 1.00 (Reference) & - \\
\hline $20-29$ & $0.71(0.59,0.86)$ & $<0.001$ \\
\hline $30-39$ & $1.26(0.99,1.61)$ & 0.06 \\
\hline $40-$ & $1.81(1.33,2.46)$ & $<0.001$ \\
\hline \multicolumn{3}{|l|}{ Education } \\
\hline Primary or less & $6.45(5.10,8.15)$ & $<0.001$ \\
\hline Junior high school & $2.54(2.06,3.13)$ & $<0.001$ \\
\hline Senior high school and above & 1.0 (Reference) & - \\
\hline \multicolumn{3}{|l|}{ Marital status } \\
\hline Never married & 1.0 (Reference) & - \\
\hline Married & $1.86(1.58,2.18)$ & $<0.001$ \\
\hline Separated/divorced/widowed & $2.15(1.54,3.01)$ & $<0.001$ \\
\hline \multicolumn{3}{|l|}{ Ethnicity } \\
\hline Han & 1.0 (Reference) & - \\
\hline Minority & $1.29(1.08,1.55)$ & 0.005 \\
\hline \multicolumn{3}{|l|}{ Household registration (hukou) } \\
\hline Local resident & $2.11(1.86,2.40)$ & $<0.001$ \\
\hline Not local resident & 1.0 (Reference) & - \\
\hline
\end{tabular}

$A O R$ adjusted odds ratio, $\mathrm{Cl}$ confidence interval.

indicated that the prevalence of visiting FSWs in the previous year was $4.2 \%$ overall, with $7.2 \%$ in urban areas and $1.8 \%$ in rural areas [20]. Although we do not have data on the percentages of Chinese men who visit each of the different tiers of FSWs, it has been found from observations in several areas that the clients of low-tier FSWs are usually migrants and elder men. It is known from our study that there are significant differences in terms of socio-demographic characteristics between different tiers of FSWs, indicating that low-tier FSWs tend to be older women with low education levels, and the women from local areas and non-Han minorities. These differences are important determinants for designing surveillance and intervention programs. The low-tier subgroups of FSWs are less well represented in current HIV and STI surveillance programs and are also less likely to be covered by current intervention programs in China. The significant differences in terms of demographic characteristics and prevalence of syphilis between FSWs recruited from low-tier sex work venues and those from middle or high-tier venues warrants consideration of what kind of population we should recruit and/or where we should recruit them to represent the FSWs for surveillance purposes. However, the interpretation of epidemiological and behavioral data emerging from such settings is challenging due to significant selection bias. The high prevalence of the syphilis infections in the study populations further emphases the 
importance of effective curative and preventive STI services for the FSW population. Syphilis surveillance and monitoring of the response to the STI epidemic may be improved by carefully considering and selecting appropriate target populations to represent women at high risk.

The strengths of this study include (1) the inclusion and classification of different tiers of FSWs in the study; (2) the utilization of community-based or outreachbased recruitment strategies; and (3) the multi-site survey consisting of 8 middle-size cities in China. There are also limitations to be addressed. First, although the study was carried out in eight cities, the socio-economic and behavioral characteristics of FSWs in the study areas may be different from other Chinese cities. Any generalization of the results from this study should therefore be made with caution. Second, the study was not able to use a random sampling method to recruit the FSWs, likely resulting in the sample bias. Third, high rates of FSWs to refuse participating in the study (i.e., 8-12\%, 20-25\% and 30-40\% among HT-, MT- and LT-FSWs, respectively) may result in potential selection bias. Fourth, the sample size for this study may not be large enough for the subgroup analysis to show potential associations between the infections and some risk factors. Fifth, this study included only limited information on risk behaviors, and data regarding sexual behaviors and condom use is subject to self-reporting bias and social response bias due to the sensitivity of these topics. Finally, this study did not ascertain an association between syphilis and HIV infection because the number of FSWs with HIV infection was small, although some studies in China have shown that HIV infection is independently associated with syphilis infection [20]. In addition, some potential factors related to the infection, such as condom use with regular non-client sexual partners and the number of clients per week, were not included in the study.

As FSWs are an important source of infection and probably the bridge population for the heterosexual transmission of syphilis and HIV, the findings of this study have a number of important implications. First, this study shows a substantial prevalence of syphilis infection among FSWs and much higher prevalence among LT- and MT-FSWs. The patterns of infection suggest the importance of prioritizing the MT- and LTFSWs in the current program for interventions (health education, condom promotion and STI care services) in FSWs. Second, this study suggests a re-appraisal of the current HIV/STI surveillance system in terms of sampling of women at high risk in China to enable more representative, valid and reliable prevalence estimates to be generated. Third, this study highlights our limited knowledge of the epidemiology of syphilis and likely other STIs among LT-FSWs in China. LT-FSWs are thought to be a potential and key co-factor for sustaining the spread of STIs in high-risk populations and for bridging the epidemic from high-risk groups to the general population. However, they are usually neglected by routine intervention programs. This underscores the urgency of including this neglected population in preventive interventions for reducing STI infection. The current HIV/STI intervention programs for female sex workers are urgently needed to improve for increasing access to MT- and LT-FSWs in China through providing a comprehensive intervention package. The package should include not only health education materials and condoms but also STI and reproductive health care including syphilis testing and counseling; and delivery of the package should be conducted by not only the solitary CDC outreach teams but also medical personnel and non-governmental organizations.

\section{Conclusions}

A substantial prevalence of syphilis in FSWs, particularly in low-tier FSWs, is a serious public health concern. Syphilis control in this population through universal screening of syphilis and integrating syphilis testing into VCT services, STI risk reduction education, and aggressive condom promotion programs are urgently needed. Much can and should be done to stem the syphilis epidemic and potential uptake of the co-epidemic of syphilis and HIV in FSWs. The Chinese Ministry of Health has already issued a national 10-year plan for syphilis control and prevention, where strengthening the surveillance system in high risk groups as well as comprehensive interventions among FSWs are priorities in order to curb the epidemic in China.

\section{Acknowledgements}

The study was supported by a grant from the Mega Project of China National Science Research for the 11th Five-Year Plan (2008ZX10001-005 to BXW). The authors would like to thank the staff who worked in the study sites for their recruiting the participants, collecting the specimens and conducting the interviews. We are also very grateful to all participants of this study for their cooperation. The authors thank Dr. Jing Li for her assistance in data analysis and Ms. Crystal Shen for her assistance in English language editing.

\section{Author details}

${ }^{1}$ National Center for STD Control, Chinese Academy of Medical Sciences and Peking Union Medical College Institute of Dermatology, 12 Jiangwangmiao Street, Nanjing 210042, China. ${ }^{2}$ Guangdong Provincial Center for Skin Diseases and STD Control, Guangzhou, China. ${ }^{3}$ Hainan Provincial Institute of Dermatology, Haikou, China. ${ }^{4}$ Guangxi Center for Diseases Control and Prevention, Nanning, China. ${ }^{5}$ Jiangsu Center for Disease Control and Prevention, Nanjing, China.

\section{Authors' contributions}

XSC, YPY, QQW, GJL, NJ and BW and conceived the study and participated in study design. LGY, QL, YJZ and XPH participated in data collection and interpretation. WHW coordinated the laboratory testing of syphilis infection and helped to interpret the data. XSC and NJ performed the statistical 
analysis. XSC and YPY prepared the manuscript, and NJ, QQW and BW helped to draft the manuscript. All authors read and approved the final manuscript.

\section{Competing interests}

The authors declare that they have no competing interests.

Received: 3 September 2011 Accepted: 4 April 2012

Published: 4 April 2012

\section{References}

1. Chen ZQ, Zhang GC, Gong XD, Lin C, Gao X, Liang GJ, Yue XL, Chen XS, Cohen MS: Syphilis in China: results of a national surveillance programme. Lancet 2007, 369:132-138.

2. Yang H, Li X, Stanton B, Liu H, Liu H, Wang N, Fang X, Lin D, Chen X: Heterosexual transmission of HIV in China: a systematic review of behavioral studies in the past two decades. Sex Transm Dis 2005, 32:270-280.

3. Wang N, Wang L, Wu Z, Guo W, Sun X, Poundstone K, Wang Y: Estimating the number of people living with HIV/AIDS in China: 2003-09. Int J Epidemiol 2010, 39(Suppl 2):ii21-ii28.

4. Powers KA, Poole C, Pettifor AE, Cohen MS: Rethinking the heterosexual infectivity of HIV-1: a systematic review and meta-analysis. Lancet Infect Dis 2008, 8:553-563.

5. Wang L, Ding ZW, Ding GW, Guo W, Wang L, Qin QQ, Li DM, Wang LY, Yan RX, Hei FX: Data analysis of national HIV comprehensive surveillance sites among female sex workers from 2004 to 2008. Zhonghua Yu Fang Yi Xue Za Zhi 2009, 43:1009-1015.

6. Chen XS, Peeling RW, Yin YP, et al: The epidemic of sexually transmitted infections in China: Implications for control and future perspectives. BMC Med 2011, 9:111.

7. In Guidelines for Laboratory Diagnosis of Sexually Transmitted Diseases. Edited by: Yin YP. Shanghai: Shanghai Science and Technology Publishing House; 2007:.

8. Lu F, Wang N, Wu Z, Sun X, Rehnstrom J, Poundstone K, Yu W, Pisani E: Estimating the number of people at risk for and living with HIV in China in 2005: methods and results. Sex Transm Infect 2006, 82(Suppl 3): iii87-iii91.

9. Choi $\mathrm{KH}$, Zheng $\mathrm{X}$, Zhou $\mathrm{H}$, et al: Treatment delay and reliance on private physicians among patients with sexually transmitted diseases in China. Int J STD AIDS 1999, 10:309-315.

10. Lin SC, Ma YG, Liao $Q$, et al: A study on relationship between first visitingdoctor behaviors of socio-demographic characteristics of STD patients. Chin J STD AIDS Prev Control 2002, 8:162-163.

11. Huang Y, Henderson GE, Pan S, Cohen MS: HIV/AIDS risk among brothelbased female sex workers in China: assessing the terms, content, and knowledge of sex work. Sex Transm Dis 2004, 31:695-700.

12. Zhou ZG, Wang H, Wang B, Zhu BY, Gan Q: A survey of behavioral characteristics and prevalence of STls among 480 female sex workers in Guangxi. Chin J Health Edu 2010, 26:522-524.

13. Yang $\mathrm{P}$, Wang $\mathrm{QQ}$, Peng H: A survey of syphilis and HIV infection in medium-low income female sex workers. China J Lepr Skin Dis 2009, 25:174-176.

14. Yang C, Latkin C, Luan R, Wang C, Nelson K: HIV, syphilis, hepatitis C and risk behaviors among commercial sex male clients in Sichuan province, China. Sex Transm Infect 2010, 86:559-564.

15. Liao M, Jiang Z, Zhang X, Kang D, Bi Z, Liu X, Fu J, Zhang N, Mao W, Jiang B, Jia YJ: Syphilis and methamphetamine use among female sex workers in Shandong Province, China. Sex Transm Dis 2011, 38:57-62.

16. Chang H, Zhi X, Chen X-S, Cohen MS: Systematic review and meta-analysis of syphilis seroprevalence among female sex workers in China Bethesda: $\mathrm{NIH}$ Fogarty International Clinical Scholar Conference; 2010.

17. Wang Q, Yang P, Gong XD, Jiang J, Yang B: Syphilis prevalence and high risk behaviors among female sex workers in different settings. China J AIDS STDs 2009, 15:398-400.

18. Yang X, Xia G: Gender, work, and HIV risk: determinants of risky sexual behavior among female entertainment workers in China. AIDS Educ Prev 2006, 18:333-347.

19. Yang X, Xia G, Li X, Latkin C, Celentano D: Social influence and individual risk factors of HIV unsafe sex among female entertainment workers in China. AIDS Educ Prev 2010, 22:69-86.
20. Lu F, Jia Y, Sun X, et al: Prevalence of HIV infection and predictors for syphilis infection among female sex workers in southern China. Southeast Asian J Trop Med Public Health 2009, 40:263-272.

\section{Pre-publication history}

The pre-publication history for this paper can be accessed here: http://www.biomedcentral.com/1471-2334/12/84/prepub

doi:10.1186/1471-2334-12-84

Cite this article as: Chen et al:: Prevalence of syphilis infection in different tiers of female sex workers in China: implications for surveillance and interventions. BMC Infectious Diseases 2012 12:84.

\section{Submit your next manuscript to BioMed Central and take full advantage of:}

- Convenient online submission

- Thorough peer review

- No space constraints or color figure charges

- Immediate publication on acceptance

- Inclusion in PubMed, CAS, Scopus and Google Scholar

- Research which is freely available for redistribution

Submit your manuscript at www.biomedcentral.com/submit
Ciomed Central 